\title{
MICROALBUMINURIA IN ESSENTIAL HYPERTENSION AND ITS RELATIONSHIP TO TARGET ORGAN DAMAGE
}

\author{
Swathy Surendran Nair', Mridulkumar Kannath ${ }^{2}$
}

${ }_{1}^{1}$ Senior Resident, Department of General Medicine, Government Medical College, Kottayam, Kerala, India.

${ }^{2}$ Associate Professor, Department of General Medicine, Government Medical College, Kottayam, Kerala, India.

\begin{abstract}
BACKGROUND
ABSTRACT diffuse endothelial dysfunction.

Aims and Objectivesand other coronary risk factors.

3. To look for evidence of target organ damage.

4. To study the relation between microalbuminuria and the indices of target organ damage.
\end{abstract}

Hypertension is a disease that increases the risk for development of cerebral, cardiac and renal events. Many patients with essential hypertension may present with overt or subclinical target organ damage at the time of their initial diagnosis. The cost effectiveness of BP reduction using drug therapy is greater in the presence of target organ abnormalities and/ or co-morbidities. Assessment of subclinical target organ damage has become the key element in evaluating hypertensive patients. Microalbuminuria is one of the earliest indications of kidney injury in patients with diabetes mellitus and hypertension and is associated with high incidence of cardiovascular morbidity. A renewed interest in microalbuminuria and essential hypertension occurred when several studies pointed out the importance of microalbuminuria as a risk factor for renal and cardiovascular disease in patients with diabetes mellitus and hypertension. It possibly reflects a state of increased renal endothelial permeability and is an early marker of

1. To study the relative frequency of microalbuminuria in essential individuals suffering from essential hypertension.

2. To study the relationship between microalbuminuria, and age of the patient, duration of hypertension, levels of blood pressure

\section{MATERIALS AND METHODS}

A total of 150 patients admitted in Government Medical College, Kottayam, Kerala with essential hypertension were studied. Detailed history regarding onset and duration of hypertension, drug treatment and symptoms of target organ involvement was taken. Detailed clinical examination and necessary investigations were done. Microalbuminuria was estimated by immunoturbidimetry method on the urine sample collected over 24 hours.

\section{RESULTS}

The prevalence of microalbuminuria was $26.7 \%$. Distribution of the end organ complications in patients with microalbuminuria were LVH in 59.1\%, Stroke in $52.4 \%$ and Retinopathy in $34.7 \%$. This difference was found to be highly significant. Microalbuminuria was found to be more prevalent in patients with longer duration, greater severity of hypertension and dyslipidaemia.

\section{CONCLUSION}

Control of risk factors amenable to prevention (control of hypertension, weight and lipid levels) may have a favourable effect in preventing, delaying and lessening microalbuminuria. Microalbuminuria in hypertensive subjects seems to be a very important test to be considered in the evaluation of target organ damage.

\section{KEY WORDS}

Microalbuminuria; Essential Hypertension.

HOW TO CITE THIS ARTICLE: Nair SS, Kannath M. Microalbuminuria in essential hypertension and its relationship to target organ damage. J. Evolution Med. Dent. Sci. 2018;7(36):3929-3934, DOI: 10.14260/jemds/2018/879

\section{BACKGROUND}

Hypertension is a disease, which affects 1 billion individuals worldwide. Recognition of microalbuminuria stemmed from Diabetes research four decades ago. According to National Kidney Foundation, Microalbuminuria is defined as a Urine Albumin Excretion Rate (UAER) of approximately 30-300 $\mathrm{mg} / \mathrm{d}$ in at least two of three consecutive samples of non-

'Financial or Other Competing Interest': None.

Submission 26-07-2018, Peer Review 21-08-2018,

Acceptance 27-08-2018, Published 03-09-2018.

Corresponding Author:

Mridulkumar Kannath,

Shreyas Mudappattuthazham,

Karuvissery P. O., Kozhikode-673010, Kerala, India.

E-mail: mridulkumar.k@gmail.com

DOI: $10.14260 /$ jemds $/ 2018 / 879$

\section{(c) $\bigcirc$}

ketotic sterile urine. In 1976, Parving et al(1) highlighted the relation between microalbuminuria and the severity of hypertension. In late 1980s, several studies pointed out the importance of microalbuminuria as an important risk factor for renal and cardiovascular disease in patients with diabetes and hypertension. Microalbuminuria possibly reflects a state of increased renal endothelial permeability and is an easily measured marker of rather diffuse endothelial dysfunction, low-grade inflammation and vascular disease burden.

\section{Aims and Objectives}

1. To study the relative frequency of microalbuminuria in individuals suffering from essential hypertension.

2. To study the relationship between microalbuminuria, and age of the patient, duration of hypertension, levels of blood pressure and other coronary risk factors. 
3. To look for evidence of target organ damage in hypertensive patients.

4. To study the relation between microalbuminuria and the indices of target organ damage.

\section{MATERIALS AND METHODS}

\section{Study Design}

A cross-sectional study.

\section{Study Location}

This study was conducted in OP and IP wing of Department of General Medicine. The study population were the patients admitted in general medicine wards and those attending medical outpatient unit in Government Medical College, Kottayam, Kerala with a diagnosis of essential hypertension.

\section{Sample Size}

Sample size for the study $=150$

Formula used: Sample size $=(\mathrm{Z})^{2} \mathrm{pq} / \mathrm{d}^{2}$

Where, $\mathrm{Z}=\mathrm{Z}$ value

$\mathrm{Z}$ value at $95 \%$ confidence interval $=1.96 ; \mathrm{Z} 2=3.84$ rounded to 4

$\mathrm{p}=$ prevalence of microalbuminuria in hypertensive $-33.3 \%$

The value of ' $p$ ' has been taken from the study by Ravjitkaur et al. Incidence of microalbuminuria in hypertensive patients, Indian journal of biochemistry 2008/23(1)71-75

$\mathrm{q}=100-\mathrm{p}$

$\mathrm{d}=$ absolute precision (8)

Therefore, sample size $=(4 \times 33.3 \times 66.7) / 8 \times 8=138$

\section{Period of Study}

11th August 2016 to 11th August 2017 (12 months).

\section{Inclusion Criteria}

Patients newly diagnosed with essential hypertension according to JNC VII criteria-

- $\quad$ Stage 1: Systolic BP of 140 to $159 \mathrm{mmHg}$ and diastolic $\mathrm{BP}$ of 90 to $99 \mathrm{mmHg}$.

- $\quad$ Stage 2: Systolic BP > $160 \mathrm{mmHg}$ and diastolic BP > 100 $\mathrm{mmHg}$.

Past history of essential hypertension.

\section{Exclusion Criteria}

1. Patients with Diabetes Mellitus: Either history of diabetes or those newly detected (Diagnosed by ADA criteria).

2. Patients with secondary hypertension.

3. Pregnancy.

4. Patients with history of ischaemic heart disease.

5. Patients with urinary tract infection by history or investigations.

6. Patients with raised serum creatinine $[>1.2 \mathrm{mg} / \mathrm{dL}]$ and macroalbuminuria (Dipstick positive albuminuria).

7. Patients on ACE inhibitors and ARBs.

After obtaining clearance from the Institutional Review Board, this study was conducted in the patients admitted and those attending medical outpatient unit under the Department of General Medicine. A total of 150 subjects were selected after attaining their consent in written format. Data collection was by clinical history, examination and investigations. A preset proforma was used to collect data.
Detailed clinical examination was done. The investigations collected were FBS, PPBS, FLP, serum creatinine, urine routine, urine culture, 24-hr urine albumin excretion, chest xray, ECG, echocardiogram in all cases and CT brain in relevant cases.

\section{Statistical Analysis}

Data analysis was done with the help of SPSS version 16. Tables and Graphs were created with the help of SPSS and Microsoft Excel. Descriptive data that included numbers and percentages were calculated for all the categories. Categorical data were analysed by Chi-square tests for statistical significance. A p-value (two-tailed) of $<0.05$ was considered statistically significant.

\section{RESULTS}

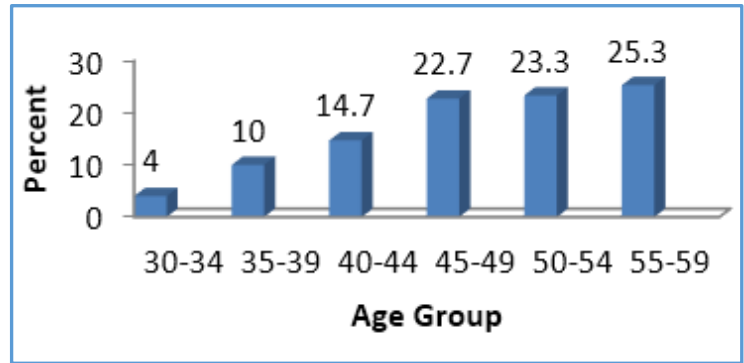

Graph 1. Distribution in different Age Groups

The minimum age was 31 and the maximum was 59 . The mean age was 48.62, SD 7.44.

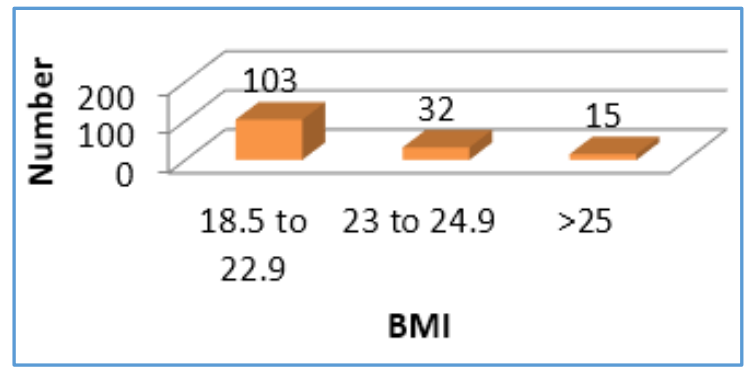

Graph 2. Distribution as per BMI

Of the total 150 subjects, $103(68.7 \%)$ belonged to the group from 18.5 to 22.9 (normal), $32(21.3 \%)$ belonged to group from 23 to 24.9 (overweight), 15 (10\%) belonged to group $>25$ (obese).

\section{Microalbuminuria}

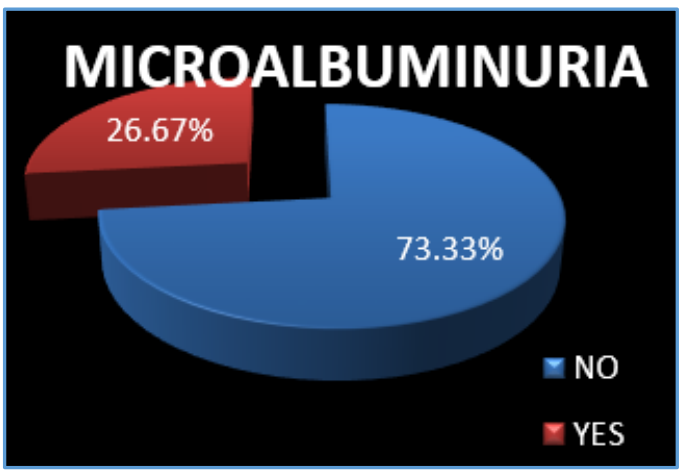

Graph 3. Proportion of Subjects with Microalbuminuria 
In this study, the number of subjects who were found to have microalbuminuria were 40 (26.7\%).

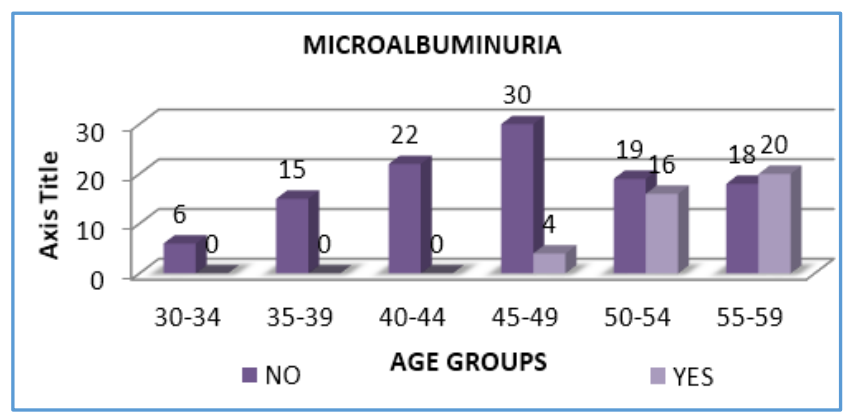

Graph 4. Distribution among different Age Groups

It was observed that as the age advances, the prevalence of microalbuminuria increases among hypertensive patients and the difference was statistically significant with $\mathrm{p}$-value $0.000(\mathrm{p}<0.05)$.

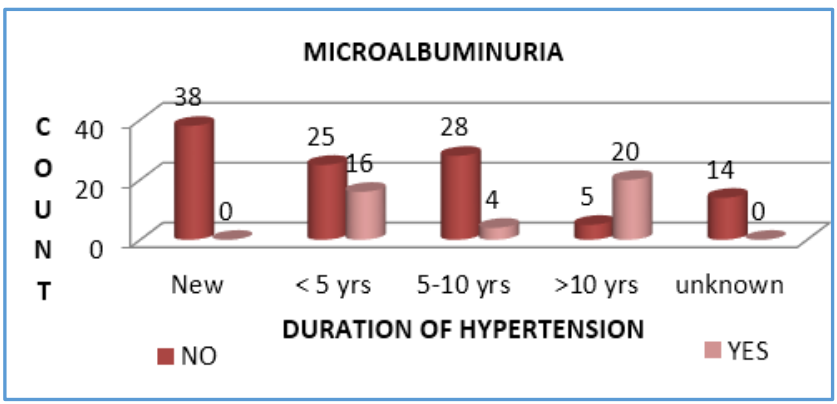

Graph 5. Microalbuminuria depending upon the Duration of Hypertension

Chi-square value 61, p-value $0.000(\mathrm{p}<0.05)$.

There was statistically significant increase in the prevalence of microalbuminuria among patients with hypertension of long duration with p-value $0.000(<0.05)$.

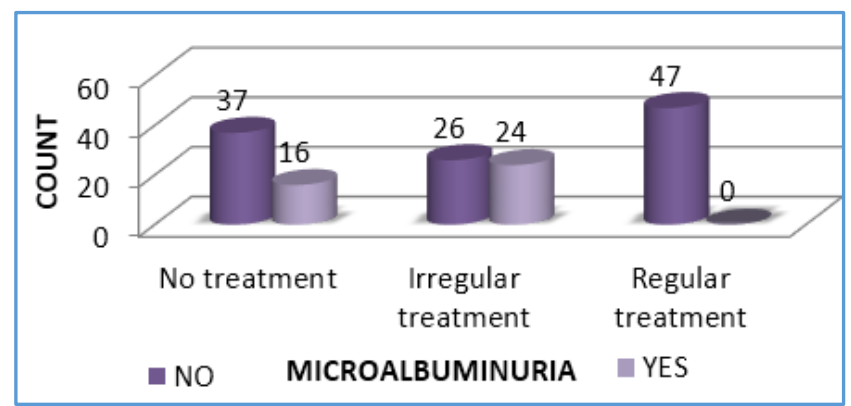

Graph 6. Microalbuminuria among Treated and Untreated Hypertensives

Among the regularly treated hypertensives, no patients had microalbuminuria and the prevalence of microalbuminuria was more in patients with no and irregular treatment and the difference was statistically significant with p-value $0.000(<0.05)$.

\section{Microalbuminuria and Lipid Profile}

Microalbuminuria was more common in those hypertensives with dyslipidaemia with a significant p-value of $0.01(<0.05)$.
Microalbuminuria and Levels of Blood Pressure

Patients with higher levels of blood pressure had higher probability of having microalbuminuria with a significant $\mathrm{p}$ value $0.003(<0.05)$.

Microalbuminuria and Indices of Target Organ Damage The indices studied were hypertensive retinopathy, stroke and abnormalities in LV geometry (LVH) and LV function.

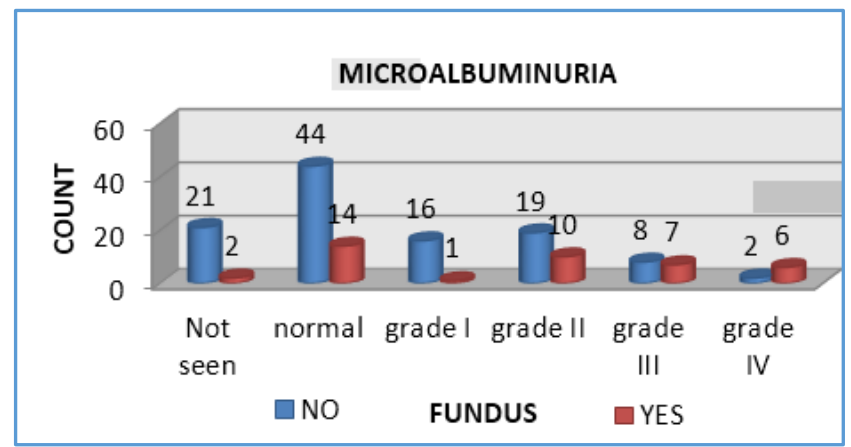

Graph 7. Microalbuminuria and Fundus

A higher proportion of patients with advanced hypertensive retinopathy had microalbuminuria and the association were statistically significant with p-value 0.001 $(<0.05 \%)$.

Stroke and Microalbuminuria

CT brain was taken for 49 patients with clinical features of stroke.

\section{CT Findings}

Normal - 7 Patients.

Abnormal - 42 Patients.

Haemorrhage - 15 Patients.

Infarct $\quad-27$ Patients.

A higher proportion of patients with hypertensive vascular involvement of brain had microalbuminuria and the association was statistically significant with $p$-value $0.01(<0.05)$.

Microalbuminuria and Hypertensive Heart DiseaseAbnormalities in LV Geometry Left Ventricular Hypertrophy [LVH]:

A total of 44 patients out of the 150 (29\%) had LVH.

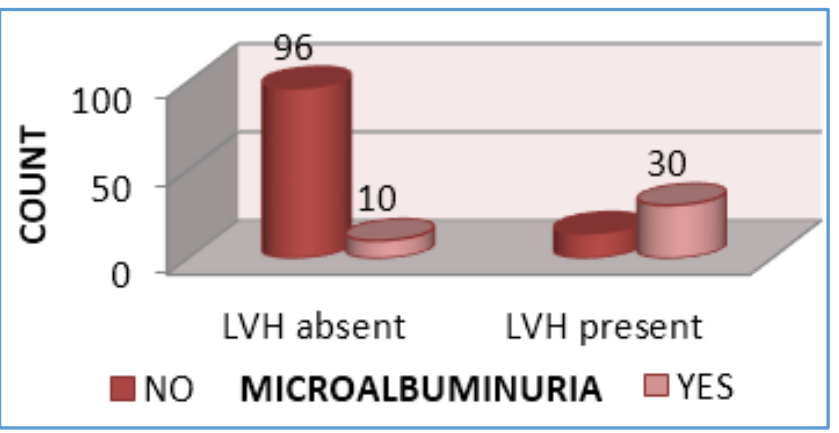

Graph 8. Microalbuminuria and LVH 
Among the patients with evidence of LVH, a significantly higher proportion had microalbuminuria with p-value 0.000 $(<0.05)$.

\section{Microalbuminuria and LV Function}

Of the total 150 subjects 15 (10\%) had LV dysfunction, 12diastolic dysfunction, 3- both systolic and diastolic dysfunction. Among the patients with LV dysfunction, prevalence of microalbuminuria was higher, and the difference was statistically significant $(\mathrm{p}<0.05 \%)$.

\section{Distribution of Microalbuminuria}

40 out of the 150 patients had microalbuminuria; $26.67 \%$.

\begin{tabular}{|c|c|}
\hline End Organ Complication & Percentage \\
\hline LVH & 59.1 \\
\hline Stroke & 52.4 \\
\hline Retinopathy & 34.7 \\
\hline $\begin{array}{c}\text { Distribution of the End Organ Complications in patients } \\
\text { with Microalbuminuria were As Follows }\end{array}$ \\
\hline
\end{tabular}

\begin{tabular}{|c|c|c|c|}
\hline & P value & Odds Ratio & 95\% CI \\
\hline LVH & $<0.001$ & 20.1 & $8.1-50.02$ \\
\hline Stroke & $<0.001$ & 3.8 & $1.8-8$ \\
\hline $\begin{array}{c}\text { Retinopath } \\
\text { y }\end{array}$ & $<0.001$ & 9.7 & $1.8-34.6$ \\
\hline \multicolumn{3}{|c|}{$\begin{array}{c}\text { Strength of association between End Organ } \\
\text { Complications in patients with Microalbuminuria }\end{array}$} \\
\hline
\end{tabular}

\section{DISCUSSION}

The present study evaluated the relationship of microalbuminuria with the indices of target organ damage in essential hypertensives; relationship between microalbuminuria and age of the patient; duration and severity of hypertension and other cardiovascular risk factors like smoking, dyslipidaemia and obesity.

\section{Prevalence of Microalbuminuria}

The prevalence of microalbuminuria in this study was $26.7 \%$ (40 out of 150 patients). In an Indian study conducted by Ravjitkaur et al in 2008, prevalence of microalbuminuria in hypertensive was 33.3\%. In 1992, Stefano Bianchi et al published the first large study on the prevalence of microalbuminuria in hypertensives. It was found to be 35\%.(1) Palatini et al in HARVEST study and the PREVEND-IT (Prevention of Renal and Vascular End Stage Disease- Study performed in the Dutch city of Groningen) showed a prevalence of 8 - 15\%. Another study by Tsioufis et al in 2002 reported a prevalence of $47 \%$. The variability in prevalence may be explained by-

- Difference in the methods of urine collection.

- Characteristics of study population.

\section{Microalbuminuria and Gender}

In the present study the number of subjects with microalbuminuria were 40 , of this 24 were males and 16 were females. The number of subjects without microalbuminuria were 110 , of this 77 were males and 33 were females and there was no statistically significant difference between the prevalence of microalbuminuria in men and women [ $\mathrm{p}=0.325]$. In the HUNT study (Norway), a stronger association was observed between microalbuminuria and mortality in men than in women. In this study, the interaction between sex and Albumin Creatinine Ratio (ACR) was statistically significant $(p=0.003)$ and supported a gender difference. Women had more prevalence. They attributed this difference to the higher incidence of asymptomatic UTI in women. They suggested the need for different ACR cut-off values in men and women, because men have greater muscle mass and higher creatinine excretion than women, although albumin excretion levels are equal. (HUNT- Nord Trondelag Health Study). Pontremoli et al(2) in the MAGIC study on the prevalence and clinical correlates of microalbuminuria observed that microalbuminuria was more common in males.

\section{Microalbuminuria and Age}

In the present study, the prevalence of microalbuminuria was higher in the older age groups $[p<0.05]$. This is in concurrence with study by Nakamura et al.(3)

\section{Microalbuminuria and Duration of Hypertension and Stages of Hypertension}

In the present study, there was statistically significant increase in the prevalence of microalbuminuria among patients with hypertension of long duration with $\mathrm{p}$-value $0.000(<0.05)$. This is in concordance with previous study by Aher et al.(4)

In the present study, patients with higher levels of blood pressure had higher probability of having microalbuminuria with a significant $p$-value $0.003(<0.05)$.

Pontremoli et al in 1997 in the MAGIC study have observed that degree and prevalence of microalbuminuria correlate with the height of $\mathrm{BP}$ when considering office values, even more so with 24-hour BP. Microalbuminuric patients are characterised by high BP levels. Cerasola et al and Ophsal et al had made similar observations. Data from the present study also showed that patients with hypertension of more than 5 years' duration were more prone to develop microalbuminuria.

\section{Microalbuminuria and Smoking}

In the present study, there were a total of 101 smokers (67.3\%) and 49 non-smokers (32.7\%). And prevalence of microalbuminuria was $23.7 \%$ among smokers and $32.7 \%$ among non-smokers and there was no statistically significant association between smoking and microalbuminuria [ $p=0.248]$. This observation is discordant with that seen in previous studies on this aspect. One possible explanation for this variability may be an error in the classification of smokers and non-smokers. This was done on the basis of history.

\section{Microalbuminuria and BMI}

In the present study prevalence of microalbuminuria in subjects with BMI normal, overweight and obese were $23.3 \%$, $40.6 \%$ and $20 \%$ respectively. And there was no statistically significant association observed between BMI and microalbuminuria [ $p=0.227$ ]. This observation is discordant with that seen in previous studies on this aspect. Del O' Mo et al (2003) had observed that microalbuminuria is more frequent in obese individuals. Leoncini et al had observed greater BMI in patients with microalbuminuria $(p<0.04)$.(2) 


\section{Microalbuminuria and Lipid Profile}

In the present study, prevalence of microalbuminuria in subjects with no dyslipidaemia and with dyslipidaemia was $19.3 \%$ and $38.6 \%$ respectively. Microalbuminuria was found to be more prevalent in those patients with dyslipidaemia and the association was statistically significant $(\mathrm{p}<0.05)$. This is in agreement with previous studies. Bianchi et al in 1997 had observed that hypertensive patients with microalbuminuria manifest increased serum LDL level and greater LDL/ HDL ratio when compared with patients without microalbuminuria and normotensives.(5)

\section{Microalbuminuria and Treated and Untreated Hypertensives \\ In the present study prevalence of microalbuminuria in subjects with no treatment, irregular treatment and regular treatment were $30.1 \%, 48 \%$ and $0 \%$ respectively. Microalbuminuria was found to be more prevalent in those patients with no treatment and irregular treatment and the association was statistically significant $(p=0.000)$. This is in agreement with previous studies. A Aher et al in 2011 had observed high prevalence of microalbuminuria in patients taking either irregular or no treatment as compared to patients on regular treatment, which was statistically significant $(p=0.006)$.}

\section{Relationship of Microalbuminuria with the Indices of- Target Organ Damage \\ Hypertensive Retinopathy}

In the present study 69 patients had changes of hypertensive retinopathy, 58 patients had normal fundus, in 23 patients fundus was not visualised due to hazy media. Of the 69 patients with fundus changes 24 patients (34.7\%) had microalbuminuria, 45 patients $(65.2 \%)$ did not have microalbuminuria, hence the present study showed a statistically significant association between microalbuminuria and the presence and severity of retinopathy $(p<0.05)$. The odds ratio for a microalbuminuric patients of having retinopathy was 9.7 (95\% Confidence Interval 2.8 - 34.6). Beisen et al in 1997 has observed an increased prevalence of hypertensive retinopathy in a group with persistent microalbuminuria despite adequate treatment ( $p<0.03)$. In 2002, Cerasola et al has observed a greater prevalence of retinopathy among those patients with microalbuminuria.

\section{Microalbuminuria and Stroke}

The present study included 42 patients with stroke, 27 patients with CT showing infarct and 15 patients with CT showing haemorrhage. Prevalence of microalbuminuria was higher among patients with stroke (52.4\%) and the association was statistically significant $(p<0.05)$. The odds ratio for a microalbuminuric patients of having cerebrovascular disease was 3.8 (95\%, Confidence Interval 1.8 - 8). It is well known that microalbuminuria is a predictor of ischaemic stroke due to its well-known association with carotid atherosclerosis (asymptomatic vascular events). There are many studies on the correlation between microalbuminuria and asymptomatic carotid artery diseaseassessed by carotid intima-media thickness; Nuclear magnetic resonance imaging etc. In a study by Pontremoli et al (2002), out of 279 patients studied Urine albumin excretion was positively associated with carotid atherosclerosis. Patients with increased UAE were 21 times more likely to develop increased carotid intima media thickness. Leena et al (Stroke - 1997) observed increased carotid intima media thickness in patients with microalbuminuria $(\mathrm{p}<0.01)$. In other studies, microalbuminuria has been shown to be a prognostic marker in ischaemic stroke.(6)

\section{Microalbuminuria and Abnormalities in LV Structure and Function}

It was observed that 44 out of 150 patients had LVH [29.3\%]. C. Tsioufis et al [2002] in their study observed that $21 \%$ of the 249 had LVH. LVH is reported in nearly $30 \%$ untreated hypertensives.

In the present study, prevalence of microalbuminuria in those without $\mathrm{LVH}$ and with $\mathrm{LVH}$ were $9.3 \%$ and $68.1 \%$ respectively and there was statistically significant association between the prevalence of microalbuminuria and the presence of LVH $(p<0.001)$ in hypertensive patients. In the present study of the total 150 subjects $15(10 \%)$ had LV dysfunction, 12 diastolic dysfunction, 3 both systolic and diastolic dysfunction. Among the patients with LV dysfunction, prevalence of microalbuminuria was higher and the difference was statistically significant $(\mathrm{p}<0.05 \%)$.

The odds ratio for a microalbuminuric patients of having LVH was 20 (95\% Confidence Interval: 8.1 - 50.2). This aspect has been studied by many investigators. W Kristian et al in 2002 (LIFE study) observed a higher prevalence of microalbuminuria $30 \%$ vs $9 \%$ in patients with concentric hypertrophy on Echo $(\mathrm{P}<0.0001)$. Pontremoli et al in 2002 has observed that patients with microalbuminuria were 21 times more likely to have LVH $(\mathrm{p}<0.001)$ in a study conducted on 279 patients in their institution. C. Stefanadis et al made similar observation in 2002. LVH was significantly higher in microalbuminuric patients (32\%) compared with normoalbuminuric subjects $(5 \%)(p<0.0001)$. The association between microalbuminuria and LV geometry may be due to haemodynamic or non-haemodynamic reasons. It is suggested that increased levels of ANP secreted from hypertrophic ventricles can directly cause microalbuminuria. The fact that subjects with heart failure and elevated ANP levels exhibiting microalbuminuria gives further support to this view.

The data from this study support the association between microalbuminuria and the duration of hypertension, the indices of target organ damage and dyslipidaemia.

\section{CONCLUSION}

In this study, $40(26.7 \%)$ subjects were found to have microalbuminuria.

1. It was observed that as the age advances, the prevalence of microalbuminuria increases among hypertensive patients and the difference was statistically significant $(\mathrm{p}=0.000)$.

2. Prevalence of microalbuminuria among patients with hypertension of long duration was more and the difference was statistically significant $(\mathrm{p}=0.000)$.

3. Microalbuminuria was more common in those hypertensives with dyslipidaemia with a significant $\mathrm{p}$ value of $0.01(<0.05)$. 
4. Patients with higher levels of blood pressure had higher probability of having microalbuminuria with a significant p-value $0.003(<0.05)$.

5. A higher proportion of patients with advanced hypertensive retinopathy had microalbuminuria and the association was statistically significant with $\mathrm{p}$-value $0.001(<0.05 \%)$.

6. A higher proportion of patients with hypertensive vascular involvement of brain had microalbuminuria and the association was statistically significant with $\mathrm{p}$-value $0.01(<0.05)$.

7. Among the patients with evidence of $\mathrm{LVH}$, a significantly higher proportion $(30 \%)$ had microalbuminuria with $\mathrm{p}$ value $0.000(<0.05)$.

8. Among the patients with LV dysfunction, prevalence of microalbuminuria was higher, and the difference was statistically significant $(\mathrm{p}<0.05 \%)$.

\section{Limitations of the Study}

1. The study did not compare the incidence of microalbuminuria in patients taking different classes of antihypertensive drugs.

2. The sample size in this study was small.

\section{REFERENCES}

[1] Bigazzi R, Bianchi S, Campese VM, et al. Prevalence of microalbuminuria in a large population of patients with mild to moderate essential hypertension. Nephron 1992;16(1):94-7.

[2] Leoncini G, Viazzi F, Vercelli M, et al. Metabolic syndrome and microalbuminuria predict renal outcome in non-diabetic patients with primary hypertension: the MAGIC study. Journal of human Hypertension 2012;26(3):149-56.

[3] Hara H, Kougami K, Shimokawa K, et al. Prevalence and risk factors of microalbuminuria in hypertensive patients under current medical treatment. Internal Medicine (Tokyo) 2014;53(12):1275-81.

[4] Aher A, Bhole P. Prevalence of microalbuminuria and its correlation to target organ damage in patients with essential hypertension. Vidarbha Journal of Internal Medicine 2014;17:9-13.

[5] Campese VM, Bianchi S, Bigazzi R. Hypertension, hyperlipidemia and microalbiminuria. Contibutions to Nephrology 1997;120;11-21.

[6] Ratnasabapathy Y, Lawes CM, Anderson CS. The Perindopril Protection Against Recurrent Stroke Study (PROGRESS): clinical implications for older patients with cerebrovascular disease. Drugs and Aging 2003;20(4):241-51. 